\title{
CALCIUM HYDROXYAPATITE CERAMIC USED AS A DELIVERY SYSTEM FOR ANTIBIOTICS
}

\author{
YOSHITAKA SHINTO, ATSUMASA UCHIDA, FEZA KORKUSUZ, NOBUHITO ARAKI, KEIRO ONO
}

From Osaka University Medical School, Japan

\begin{abstract}
Porous blocks of calcium hydroxyapatite ceramic were evaluated as delivery systems for the sustained release of antibiotics. We tested gentamicin sulphate, cefoperazone sodium, and flomoxef sodium in powder form placed in a cylindrical cavity in calcium hydroxyapatite blocks, using in vitro studies of elution and in vivo studies in rats.

Gentamicin sulphate gave a maximum concentration within the first week, which gradually decreased but was still effective at 12 weeks, when $70 \%$ of the antibiotic had been released. Even at this stage the antibiotic concentration from a $75 \mathrm{mg}$ dose was five times the minimum inhibitory concentration for staphylococci. In the in vivo studies the release of gentamicin sulphate into the normal bone of rats was at similar rates and levels. The bacteriocidal activity of the drugs was not affected by packing into calcium hydroxyapatite ceramic and the blocks were completely biocompatible on histology.

This new system overcomes the disadvantages of other drug delivery systems, avoiding thermal damage to the antibiotics and a second operation for the removal of the carrier. Some mechanical strength is provided by the ceramic and healing may be accelerated by bone ingrowth into its micropores.
\end{abstract}

A high concentration of antibiotics at the site of infection is useful in the treatment of chronic haematogenous or implant-associated osteomyelitis. The parenteral administration of drugs may not achieve effective local concentrations, as infected bone has an uncertain blood circulation. Several delivery systems have been used, including closed suction-irrigation (Kelly, Martin and Coventry 1970; Tong et al 1987), and materials which can retain antibiotics such as the patient's own blood (Zilch and Lambiris 1986), plaster pellets (Dahners and Funderburk 1987), fibrin (Zilch and Lambiris 1986), acrylic resin (Hanamura 1984), collagen (Ascherl et al 1986), and acrylic bone cement (Vécsei and Barquet 1981 ; Majid et al 1985; Salvati et al 1986).

Porous calcium hydroxyapatite (CHA) ceramic has excellent biocompatibility (Bhaskar et al 1971; Klawitter et al 1976; Uchida et al 1984, 1985, 1987; Eggli, Müller and Schenk 1988), can resist mechanical force (Lemons 1988) and is effective in filling bone defects after

Y. Shinto, MD, Research Fellow

A. Uchida, MD, Assistant Professor

F. Korkusuz, MD, Research Fellow

N. Araki, MD, Research Fellow

K. Ono, MD, Professor and Head of Department

Department of Orthopaedic Surgery, Osaka University Medical School,

1-1-50 Fukushima, Fukushima-ku, Osaka 553, Japan.

Correspondence should be sent to Dr A. Uchida.

(C) 1992 British Editorial Society of Bone and Joint Surgery 0301-620X/92/4382 $\$ 2.00$

J Bone Joint Surg [Br] 1992; 74-B: 600-4. resection of benign tumours (Uchida et al 1990). We have already used porous $\mathrm{CHA}$ as a slow-release delivery system for an anticancer drug, taking advantage of its interconnecting pore structure, and animal experiments (Uchida et al 1992) have confirmed its sustained-release capacity.

We now report a modification of this delivery system applied to the sustained release of antibiotics for the treatment of chronic osteomyelitis.

\section{MATERIALS AND METHODS}

Preparation of antibiotic-CHA. Porous CHA ceramic blocks of $10 \times 10 \times 10 \mathrm{~mm}$ and $4 \times 3 \times 3 \mathrm{~mm}$ (Central Glass Laboratories, Japan) were sintered at $1200^{\circ} \mathrm{C}$ for two hours. The porosity of the blocks was $30 \%$ to $40 \%$, with micropore diameters of from 40 to $150 \mu$ and the interconnecting pore structure open on the surfaces of the blocks. One of three different antibiotic powders, gentamicin sulphate (Schering, USA), cefoperazone sodium (Pfizer, USA), and flomoxef sodium (Shionogi, Japan), was packed into a central cylindrical cavity in each porous block, and the cavity sealed with $\alpha$-tricalcium phosphate glue (Fig. 1). There were $75 \mathrm{mg}$ of antibiotic in the larger blocks for the in vitro studies and $5 \mathrm{mg}$ in the smaller blocks for the in vivo studies.

In vitro studies. Six large blocks were prepared for each antibiotic. Each was placed in a Falcon tube containing $5 \mathrm{ml}$ of phosphate-buffered saline (PBS) and incubated 
at $37^{\circ} \mathrm{C}$. The PBS was replaced at 24-hour intervals for 90 days, and the removed elution fluids were preserved at $-45^{\circ} \mathrm{C}$ for later determination of the antibiotic concentrations.

In vivo studies. Albino Sprague-Dawley rats weighing 500 to $600 \mathrm{~g}$ were anaesthetised with $10 \mathrm{mg} / \mathrm{kg}$ intraperitoneal ketamine hydrochloride (Sankyo, Japan). An ethylene-oxide sterilised small block, containing $5 \mathrm{mg}$ of gentamicin sulphate, was then implanted into the proximal tibia of each rat under sterile conditions. The skin was closed by staples and the animals were then allowed free movement in their cages. The composite blocks were removed at regular intervals after killing the animals and specimens of local bone and muscle, and of plasma, kidney and liver were removed and preserved at $-45^{\circ} \mathrm{C}$. These were later homogenised with $10 \mathrm{ml}$ of PBS and the suspension used for antibiotic assay.

Antibiotic assay. Microbiological assays were performed by an agar diffusion paper-disc method using Bacillus

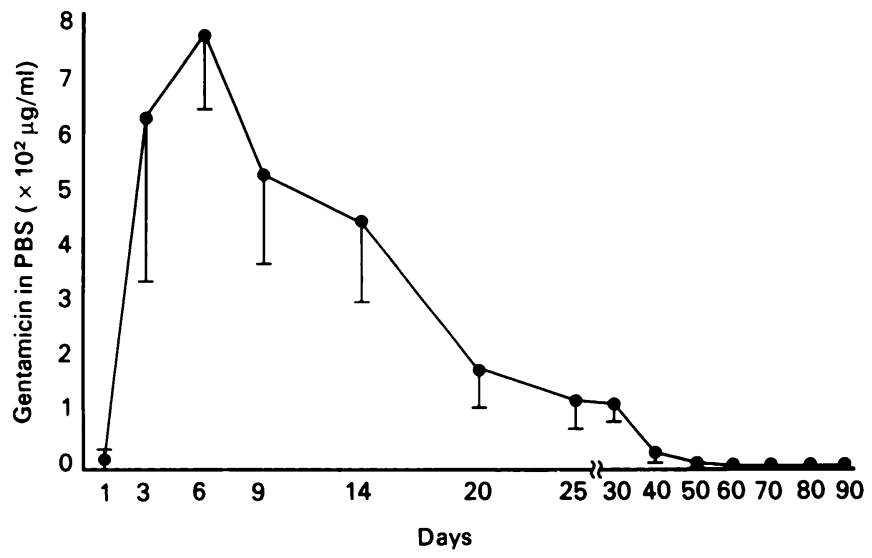

Fig. 2
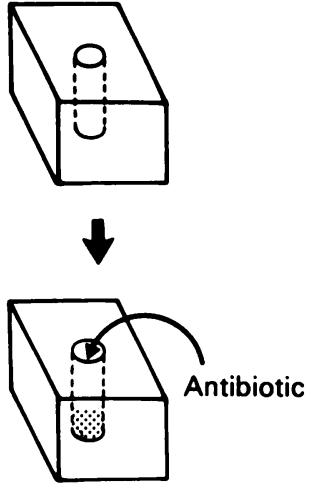

Diagrams of an antibiotic-CHA ceramic block. The small cylindrical hole is made by ultrasound, powdered antibiotics are packed into the hole and sealed in with $\alpha$ tricalcium phosphate (TCP) in polylactic acid.

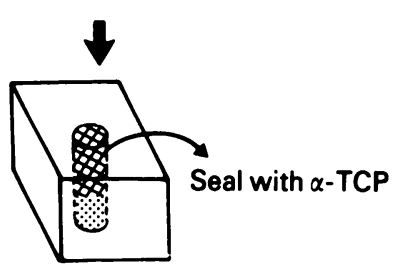

Fig. 1

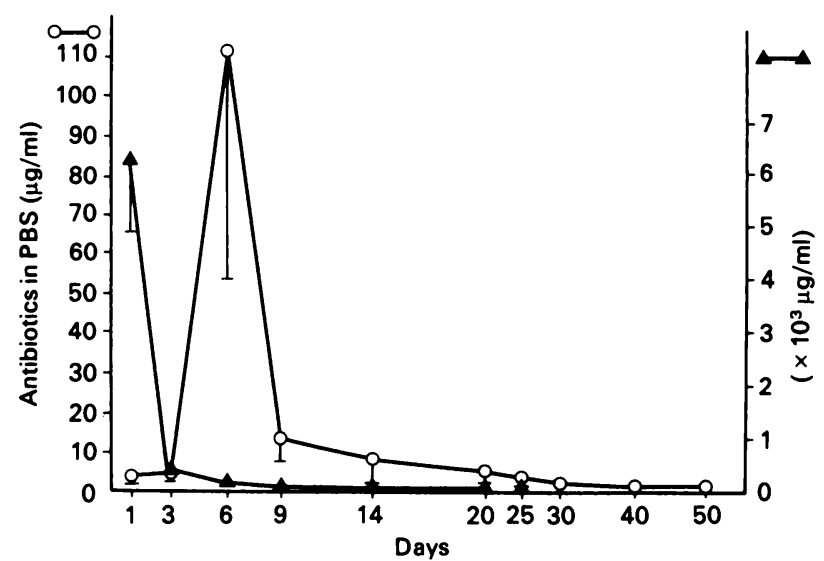

Fig. 3

Figure 2 - Gentamicin concentration in the elution fluid (mean \pm SD for six samples). Figure 3 - Levels of cefoperazone sodium (circles) and flomoxef sodium (triangles) in elution fluid (mean \pm SD for six samples).

subtilis (ATCC 6633), and the results confirmed by fluorescence polarisation immunoassay using a TDX machine (Abbott Laboratories, USA). The lowest sensitivity level for the assay was $0.05 \mu \mathrm{g} / \mathrm{ml}$.

Bacteriocidal activity. The elution fluids obtained from the in vitro study and the suspension obtained from homogenised local bone in the in vivo study were evaluated for the bacteriocidal activity of gentamicin sulphate. Two $0.1 \mathrm{ml}$ samples of each specimen were placed in the central hole of a trypticase soy agar plate containing a stock strain of Staphylococcus aureus 209p $(10 \mathrm{CFU} / \mathrm{ml})$, and incubated at $37^{\circ} \mathrm{C}$ for 24 hours. The zones of bacterial inhibition were recorded and compared with the inhibition zones for similar concentrations of freshly made gentamicin sulphate solution.

Histology. Blocks containing gentamicin sulphate were implanted into the tibiae of seven Sprague-Dawley rats to assess the biocompatibility of the implants. The animals were killed at monthly intervals and a portion of the tibia containing the implant was removed with the surrounding bone marrow and muscles. The specimen was fixed in $10 \%$ buffered formal saline, decalcified in $10 \%$ formic acid and sections were stained with haematoxylin and eosin for light-microscopic examination.

\section{RESULTS}

In vitro study. Gentamicin sulphate elution from the composite increased to reach its peak during the second week, then gradually decreased until the end of the experiment at 90 days. The mean concentration of the drug at 90 days remained at least five times higher than the minimum inhibitory concentration for staphylococci $(1.25 \mu \mathrm{g} / \mathrm{ml})$, although the composite contained only 
$75 \mathrm{mg}$ of gentamicin sulphate (Fig. 2). By 90 days, $70 \%$ of the drug had been released. Cefoperazone sodium showed a similar release pattern to gentamicin sulphate, reaching its peak on the sixth day, and gradually decreasing until 20 days, after which it was detected for 30 more days in low concentration (Fig. 3). Flomoxef sodium showed the most rapid release, with a very high concentration on the first day, but it was not detected after 25 days (Fig. 3).

In vivo study. For the in vivo experiments, we used only gentamicin sulphate since it had shown the longest release duration in the in vitro study. The release pattern from the composite into normal bone of rats was similar to that found in the in vitro study, the antibiotic concentration reaching a peak eight days after implantation, and gradually decreasing until 90 days. The concentration of gentamicin sulphate in the kidney, liver, and plasma remained extremely low in comparison with that in bone (Fig. 4).

Bacteriocidal activity. The elution fluid from the in vitro study showed a strong bacterial inhibitory effect for a long period, and was directly related to the measured concentration of the drug released at each stage (Fig. 5). This demonstrates that the antibiotic packed into the ceramic remains chemically stable and fully active after release from the composite block.

Histology. At one month after implantation there was some bone ingrowth into the micropores of the antibiotic-

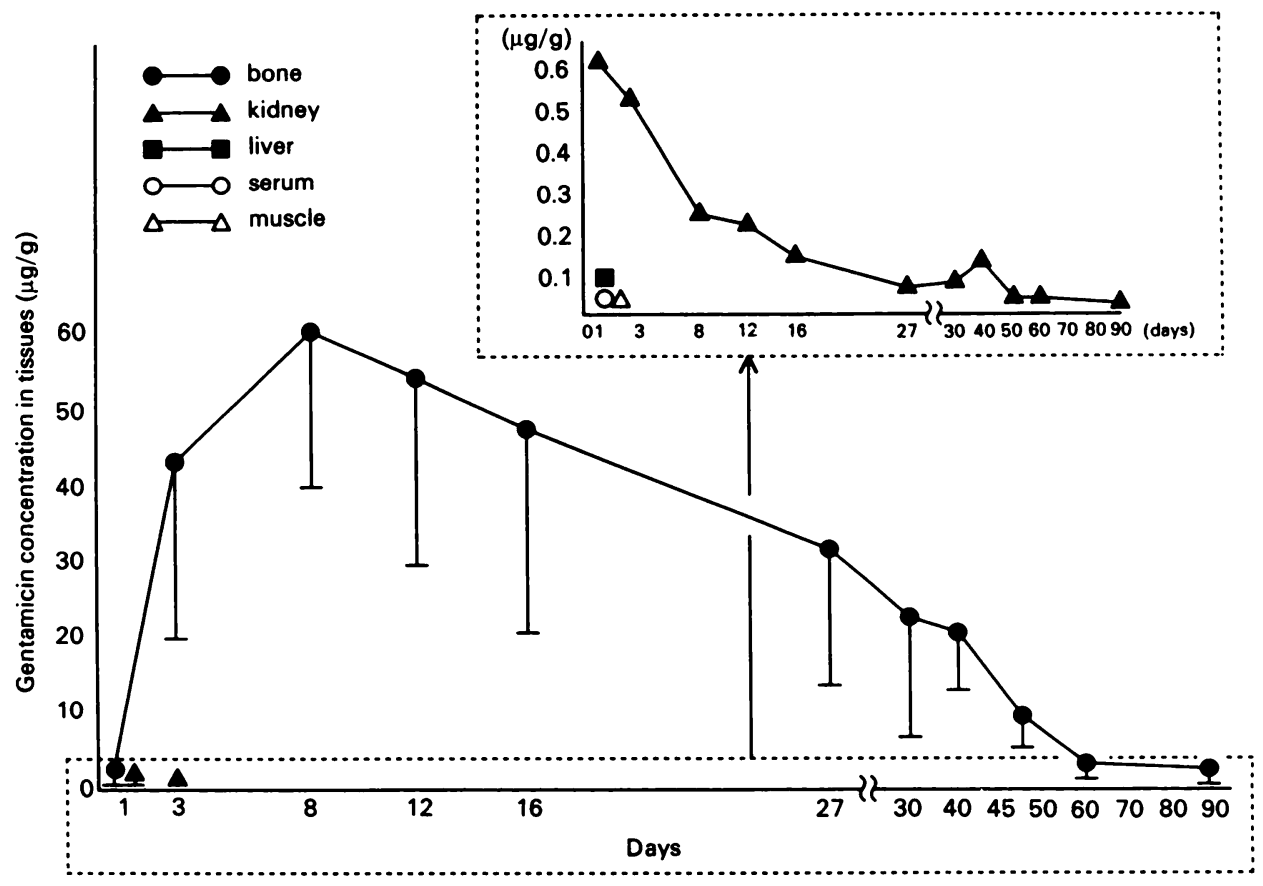

Fig. 4

Tissue and plasma concentrations of gentamicin after the implantation of a gentamicin-CHA slowrelease block into rat tibiae (mean \pm SD for six samples).

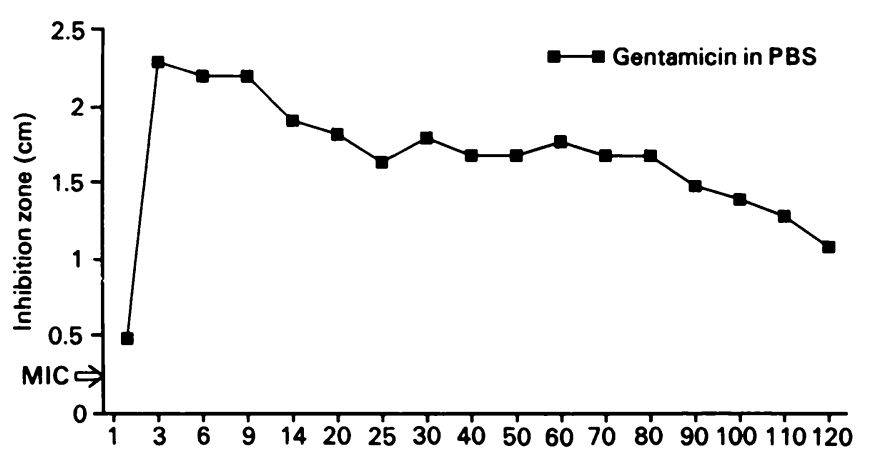

Fig. 5

Bacteriocidal activity of the gentamicin released from an antibioticCHA block into the solution corresponded to the released drug concentration shown in Figure 2.
CHA block in decalcified sections. The pores were not filled with new bone, however, until after six months, and no difference was detected between antibiotic-CHA blocks and CHA blocks, showing that packing gentamicin sulphate into a central opening did not alter the biocompatibility of the ceramic (Fig. 6).

\section{DISCUSSION}

Several biomaterials have been used as vehicles for the transport and sustained release of antibiotics. Antibioticimpregnated acrylic bone cement has been studied in detail (Chapman and Hadley 1976; Rosenthal, Rovell and Girard 1976; Whaling et al 1978; Bayston and Milner 1982; Kirkpatrick et al 1985; Baker and Green- 


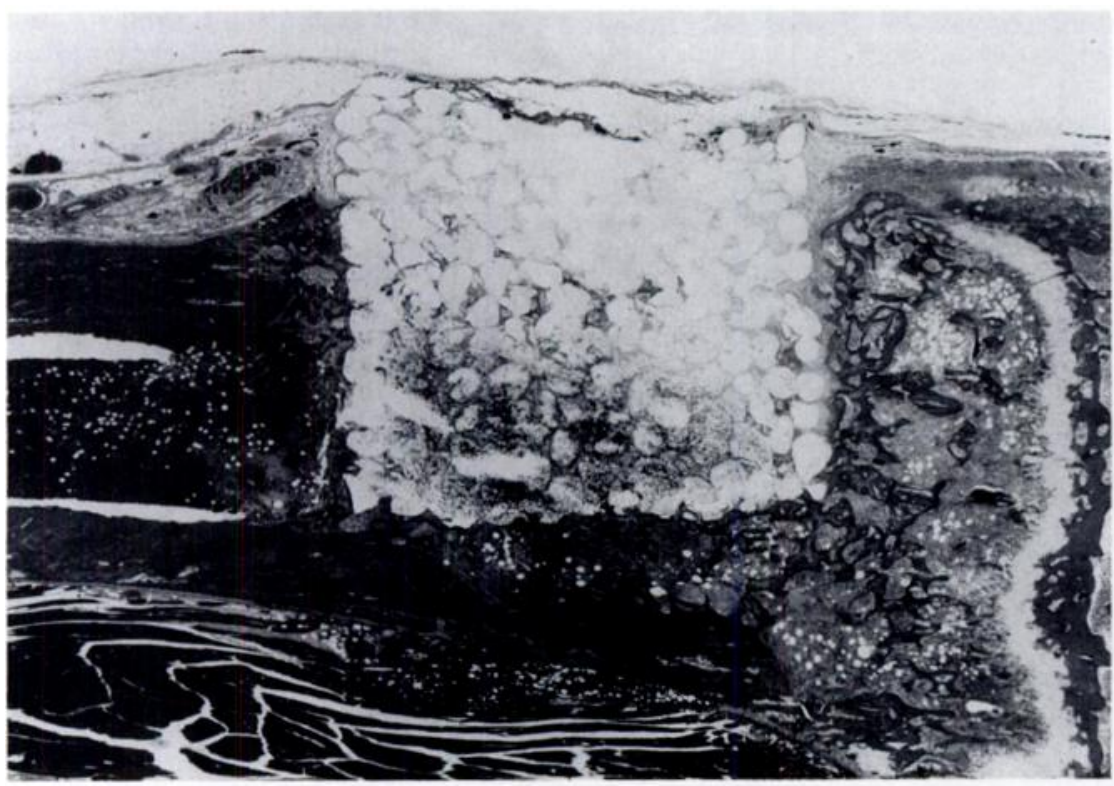

Fig. 6a

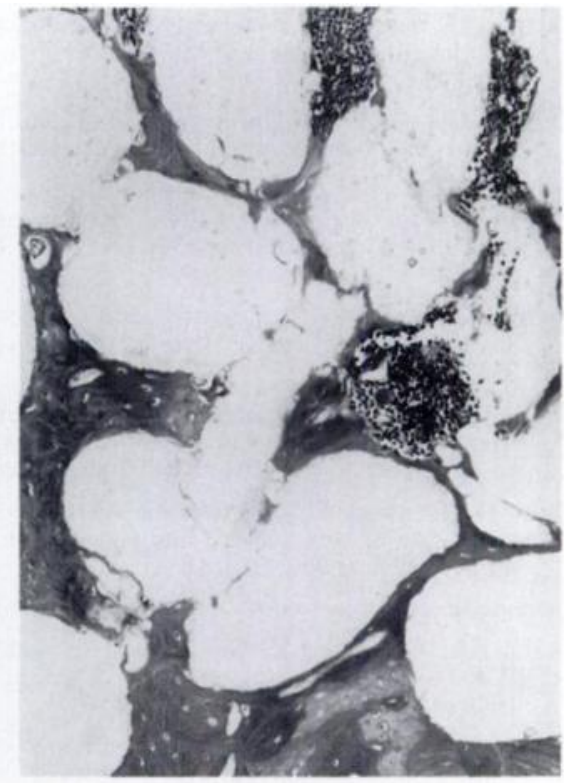

Fig. 6b

Histology six months after implantation of an antibiotic-CHA block, showing biocompatibility. Bone and bone-marrow cells have grown into the pore structure but some gentamicin particles are visible as small black granules $(\mathrm{a}, \times 15, \mathrm{~b}, \times 300)$.

ham 1988) as has its application for the treatment of chronic or implant-associated osteomyelitis. The reported disadvantages include:

1) reduced biocompatibility with bone;

2) short duration of release;

3) very low release rate; and

4) thermal damage to the antibiotics.

Recently, a biodegradable bone cement polypropylene fumarate-methylmethacrylate (PPF-MMA) has been proposed as a local drug delivery system, which overcomes some of these disadvantages (Gerhart et al 1988), although thermal damage to the antibiotics and a foreign-body reaction do still occur.

Our delivery system is superior to acrylic bone cement. Any antibiotic can be placed in CHA because there is no thermal damage to the drug. All the implanted antibiotic is released over a long period, none being trapped in the composite. CHA ceramics are fully biocompatible; bone and bone-marrow cells are seen to grow into the micropores and probably provide mechanical stability. These findings make it possible that filling the defects caused by the resection of infected bone with these composites can control infection and avoid the need for a second operation.

The different release patterns of the three antibiotics studied may be related to their solubility. Cefoperazone and flomoxef are more soluble in saline than gentamicin sulphate, but it showed the slowest release; this therefore indicates a means of controlling release rates.

In the treatment of chronic osteomyelitis, systemic administration of antibiotics for a long period may give rise to side-effects such as myelosuppression, nephrotoxicity and drug-induced hepatitis, while the side-effects of aminoglycosides are well known (Esterhai, Bednar and
Kimmelman 1986). The plasma and liver concentrations of gentamicin sulphate reached after local administration with our system were low enough to avoid these serious side-effects.

We suggest that antibiotic-CHA composites may be a useful method for the treatment of chronic haematogenous or implant-associated osteomyelitis. We propose to test its effectiveness and clinical feasibility in the treatment of implant-associated chronic osteomyelitis in an animal model.

The authors would like to thank Professor Sydney Nade, University of Sydney, Australia for valuable comments and suggestions.

No benefits in any form have been received or will be received from a commercial party related directly or indirectly to the subject of this article.

\section{REFERENCES}

Ascherl R, Stemberger A, Lechner F, et al. Behandlung der chronischen osteomyelitis mit einem kollagen-antibiotika-verbund-vorläufige mitteilung. Unfallchirurgie 1986; 12:125-7.

Baker AS, Greenham LW. Release of gentamicin from acrylic bone cement: elution and diffusion studies. $J$ Bone Joint Surg [Am] 1988: 70-A :1551-7.

Bayston R, Milner RDG. The sustained release of antimicrobial drugs from bone cement: an appraisal of laboratory investigation and their significance. J Bone Joint Surg [Br] 1982;64-B:460-4.

BhaskarSN, Brady JM, Getter L, Grower MF, Driskell T. Biodegradable ceramic implants in bone: electron and light microscopic analysis. Oral Surg Oral Med Oral Path 1971 ; 32:336-46.

Chapman MW, Hadley WK. The effect of polymethylmethacrylate and antibiotic combinations on bacterial viability: an in vitro and preliminary in vivo study. $J$ Bone Joint Surg [Am] 1976; 58-A: 76-81.

Dahners LE, Funderburk CH. Gentamicin-loaded plaster of paris as a treatment of experimental osteomyelitis in rabbits. Clin Orthop 1987; 219:278-82.

Eggli PS, Mäller W, Schenk RK. Porous hydroxyapatite and tricalcium phosphate cylinders with two different pore size ranges implanted in the cancellous bone of rabbits. Clin Orthop 1988; 232:127-38. 
Esterhai JL, Bednar J, Kimmelman CP. Gentamicin-induced ototoxicity complicating treatment of chronic osteomyelitis. Clin Orthop 1986; 209:185-8.

Gerhart TN, Roux RD, Horowitz G, et al. Antibiotic release from an experimental biodegradable bone cement. J Orthop Res 1988; $6: 585-92$.

Hanamura T. Experimental study of acrylic resin coated gentamicin tables for local antibiotic therapy. J Jap Orthop Ass 1984; 58: 555-65.

Kelly PJ, Martin WJ, Coventry MB. Chronic osteomyelitis. II. Treatment with closed irrigation and suction. JAMA 1970; $213: 1843-8$

Kirkpatrick DK, Von Fraunhofer JA, Trachtenberg LS, Seligson D, Mangino PD. In vitro characteristics of tobramycin-PMMA beads: comprehensive strength and leaching. Orthopedics 1985; 8:1130-3.

Klawitter JJ, Bagwell JG, Weinstein AM, Sauer BW, Pruitt JR. An evaluation of bone growth into porous high density polyethylene. J Biomed Mater Res 1976; 10:311-23.

Lemons JE. Hydroxyapatite coatings. Clin Orthop 1988; $235: 220-3$.

Majid SA, Lindberg LT, Gunterberg B, Siddiki MS. GentamicinPMMA beads in the treatment of chronic osteomyelitis. Acta Orthop Scand 1985; 56:265-8.

Rosenthal AL, Rovell JM, Girard AE. Polyacrylic bone cement containing erythromycin and colistin. I. In vitro bacteriological activity and diffusion properties of erythromycin, colistin and erythromycin/colistin combination. J Int Med Res 1976; 4: 296-304.
Salvati EA, Callaghan JJ, Brause BD, Klein RF, Small RD. Reimplantation in infection: elution of gentamicin from cement and beads. Clin Orthop 1986; 207:83-93.

Tong Y, Wei X, Qiu G, Ren Y, Wang GS. The treatment of chronic hematogenous osteomyelitis. Clin Orthop 1987; 215:72-7.

Uchida A, Nade SML, McCartney ER, Ching W. The use of ceramics for bone replacement. J Bone Joint Surg [Br] 1984; 66-B:269-75.

Uchida A, Nade S, McCartney E, Ching W. Bone ingrowth into three different porous ceramics implanted into the tibia of rats and rabbits. J Orthop Res 1985; 3:65-77.

Uchida A, Nade S, McCartney E, Ching W. Growth of bone marrow cells on porous ceramics in vitro. J Biom Mater Res 1987; $21: 1-10$.

Uchida A, Araki N, Shinto Y, et al. The use of calcium hydroxyapatite ceramics in bone tumour surgery. J Bone Joint Surg [Br] 1990; 72B:298-302.

Uchida A, Shinto Y, Araki N, et al. Slow release of anticancer drugs from porous calcium hydroxyapatite ceramic. J Orthop Res 1992; 10 :in press.

Vécsei V, Barquet A. Treatment of chronic osteomyelitis by necrectomy and Gentamicin-PMMA beads. Clin Orthop 1981; 159:201-7.

Whaling $\mathbf{H}$, Dingelden $\mathbf{E}$, Bergmann $\mathbf{R}$, Reuss $\mathbf{K}$. The release of gentamicin from polymethlmethacrylate beads. J Bone Joint Surg [Br] 1978; 60-B:270-5.

Zilch $\mathbf{H}$, Lambiris $\mathbf{E}$. The sustained release of cefotaxim from a fibrincefotaxim compound in treatment of osteitis. Arch Orthop Trauma Surg 1986; 106:36-41 